Pablo Galindo-Moreno

Miguel Padial-Molina

Gustavo Avila

Hector F. Rios

Pedro Hernández-Cortés

Hom-Lay Wang

\section{Complications associated with implant migration into the maxillary sinus cavity}

Authors' affiliations:

Pablo Galindo-Moreno, Oral Surgery and Implant

Dentistry Department, School of Dentistry,

University of Granada, Granada, Spain

Miguel Padial-Molina, Hector F. Rios, Hom-Lay

Wang, Department of Periodontics and Oral

Medicine, School of Dentistry, University of

Michigan, Ann Arbor, MI, USA

Gustavo Avila, Department of Periodontics,

University of Iowa College of Dentistry, Iowa City, IA, USA

Pedro Hernández-Cortés, Orthopedic Surgery Unit,

San Cecilio Clinical Hospital, Granada, Spain

Corresponding author:

Dr. Pablo Galindo-Moreno

C/Recogidas, $395^{\circ}$ Izq, 18005 Granada, Spain

Tel.: +34958 520658

Fax: +34 958520658

e-mail: pgalindo@ugr.es/pablogm@umich.edu
Date:

Accepted 11 June 2011

To cite this article:

Galindo-Moreno P, Padial-Molina M, Avila G, Rios HF,

Hernández-Cortés P, Wang H-L. Complications associated

with implant migration into the maxillary sinus cavity.

Clin. Oral Impl. Res. 23, 2012, 1152-1160

doi: 10.1111/j.1600-0501.2011.02278.x

Key words: alveolar ridge augmentation, bone grafting, complications, dental implants, maxillary sinus, migration

\begin{abstract}
Background: Migration of dental implants into the maxillary sinus is an uncommon, but increasingly reported complication. Implant migration may result from initial lack of primary stability, intrasinusal and nasal pressure changes, autoimmune reaction to the implant or incorrect distribution of occlusal forces. This retrospective study aims at analyzing the factors that may influence implant migration into the maxillary sinus cavity.

Material and methods: Fourteen patients presenting a total 15 implants that migrated into the maxillary sinus were recruited. Diagnosis of this complication was based on imaging techniques, such as cone beam computerized tomography scan and panoramic radiography. Clinical data were recorded in all cases and processed for statistical analysis.

Results: $\mathrm{ABH}$ was below $6 \mathrm{~mm}$ in the majority of cases. However, almost $50 \%$ of the patients did not receive any site preparation treatment prior to implant insertion. Five patients (33.3\%) were treated by osteotome techniques, but only one of them had bone grafting. Therefore, $73.3 \%$ of sites did not receive any biomaterial to increase available bone height. The most common complication-associated factors found on this study were related to implant design (cylindrical), implant dimension (diameter), implant restoration/rehabilitation method (partial removable denture), site-specific anatomy (initial residual bone height between 5 and $6.9 \mathrm{~mm}$ ), demographics (age), and biomaterials.

Conclusion: Patient selection and proper treatment planning, as well as the application of the appropriate sinus augmentation technique, are critical aspects that should be controlled to minimize the risk of implant migration into the maxillary sinus cavity. [Correction added after online publication August 17 2011: The Conclusion was revised to provide better clarity to the reader.]
\end{abstract}

Occlusal rehabilitation of the edentulous posterior maxilla with implant-supported restorations represents a unique clinical challenge. Posterior upper maxilla bone is typically soft, due to its thin or non-existing cortical and very spongiotic trabeculae, possibly compromising implants' primary stability and, therefore, consecutively its implant failure (Adell et al. 1990; Misch 1990a). To offset this biomechanical disadvantage different therapeutic strategies have been developed. These include, but are not limited to, special drilling protocols, modified implant designs, and the use of bone condensers (e.g. osteotome-based implant placement). On the other hand, tooth loss typically triggers a cascade of events that ultimately leads to alveolar bone resorption (Schropp et al. 2003). Resorptive processes are particularly dramatic in the posterior maxilla, resulting in marked vertical bone deficiency that may contraindicate conventional implant placement. Various therapeutic alternatives have been proposed to overcome this limitation. Sinus floor elevation, also known as sinus augmentation, is regarded as a predictable procedure for implant site development in this region. Since it was first described (Boyne \& James 1980), this technique has proven its efficacy and reliability in a variety of clinical scenarios using different grafting materials, and modifications of the original surgical protocol (Wallace \& Froum 2003; Pjetursson et al. 2008; Tan et al. 2008). A number of alternatives 
to ridge augmentation procedures, such as tilted implants, zygomatic implants, pterygoid implants, short implants $(<10 \mathrm{~mm})$, restorations in cantilever or even graftless sinus floor elevation have been described as suitable methods to restore posterior occlusal function with implant-supported prostheses (Thor et al. 2007).

An increasing debate exists in the scientific community regarding the treatment of choice to obtain satisfactory outcomes with minimal trauma and to shorten the total treatment time. However, selection of an inadequate treatment option may derive into serious complications, such as implant migration inside the sinus cavity. Since the first case was described (Regev et al. 1995), other authors have depicted the occurrence of this adverse event into the maxillary and other paranasal sinuses. Most reports have included a limited number of implants (Regev et al. 1995; Iida et al. 2000; Raghoebar \& Vissink 2003; Nakamura et al. 2004; Galindo et al. 2005; Varol et al. 2006; Guler \& Delilbasi 2007; Kim et al. 2007; Kitamura 2007; Lubbe et al. 2008; Flanagan 2009; Borgonovo et al. 2010; Kluppel et al. 2010; Ramotar et al. 2010; Scarano et al. 2010; Tsodoulos et al. 2010), with only a couple that include a slightly larger series of cases (Chiapasco et al. 2009; Ridaura-Ruiz et al. 2009). Various treatment modalities have been employed to deal with this complication, from a conservative approach (i.e. leave the migrated implant untreated under monitoring) to endoscopic transnasal procedures or a conventional Cadwell-Luc technique.

Different theories have been proposed and aimed at explaining the mechanism by which implant migrations occurs. Some of the proposed primary factors involved in this complication include changes in the intrasinusal and nasal pressures (Galindo et al. 2005), autoimmune reaction to the implant or incorrect distribution of occlusal forces (Regev et al. 1995). Nevertheless, it is important to consider that inadequate treatment to rehabilitate edentulous segments of the posterior maxilla (e.g. absence of implant site development) may be the underlying cause of implant migration in many instances (Chiapasco et al. 2009).

This retrospective study aimed at identifying the factors that may contribute to the occurrence of implant migration into the maxillary sinus cavity. In addition, we evaluated the pathology derived from these adverse events and proposed different therapeutic approaches to resolve these complications.

\section{Materials and methods}

\section{Study population}

Migrated implants from patients who suffered dental implant displacement into the maxillary sinus were included in this retrospective study. Migrations took place at different stages of the treatment sequence and maintenance, between the years 2005 and 2010. Patients were treated in a private practice setting (P.G.-M.). Institutional Review Board from the University of Michigan issued an exemption to this study because of the use of collected existing data in such a manner that subjects cannot be identified, directly or through identifiers linked to the subjects (HUM0048824). All of the patients were informed about their clinical circumstances, and everyone who underwent corrective surgery signed an informed consent.

\section{Data collection}

Diagnosis of the migration was assessed based on imaging techniques, such as cone beam computerized tomography (CBCT) and panoramic radiography (PR). Radiographic diagnosis was complemented with a clinical examination in all patients.

Standardized digital panoramic radiographs (Kodak ACR-2000; Eastman Kodak Company, Rochester, NY, USA) were obtained at the diagnosis appointment, prior to surgery when it was realized. Specialized software (Dent-A-View v1.0; DigiDent, DIT, Nesher, Israel) was used to make linear measurements.

Information recorded included patient's age and gender, smoking habits (smoker/nonsmoker), initial implant location, implant diameter and length, implant macro- and micro design features (i.e. implant design and type of surface), sinus augmentation status (presence or absence), grafting material used, available bone height at the time of implant placement $(\mathrm{ABH})$, type of prosthesis, pathology derived from the migration and type of therapy indicated to resolve the complication.

\section{Data analysis}

Statistical data analysis was aimed firstly at describing the main features of the distribution of the measures: central tendency and data dispersion for scalar variables, and relative frequencies for categorical ones. Only 14 implants were considered for the statistical analyses, considering just one implant per patient. Binomial and chi-square randomization-based tests were used for the analysis of proportions. Kendall Tau-b was used for determining the significance of associations between ordinal and scale variables, and Cramer $\mathrm{V}$ was used for pairs of nominal variables. Secondly, we studied how patient factors (smoking habits, alveolar height) and implant features (implant design, diameter, length) affect implant migration into sinus cavity. Finally, backward logistic regression ( $P$ out $=0.10,20$ interactions $)$ was used to explore whether presence or absence of related complications can be postdicted. Presence/absence of complications served as the dependent and age, gender, smoking habits, alveolar height, implant diameter, implant length, and biomaterial served as predictors. Analyses were performed using SPSS for Windows (PASW 18.0; SPSS Inc., Chicago, IL, USA).

\section{Results}

Fourteen patients (6 women) presenting a total of 15 migrated implants were enrolled in this study. One patient presented two migrations in the same maxillary sinus (Fig. 1), although just one of them was considered in the statistical analyses. Mean age was 54.87 years $(S D \pm 8.75)$, ranging from 38 to 65 years. A total of $66.7 \%$ of the subjects were smokers. Mean $\mathrm{ABH}$ was $5.2 \mathrm{~mm}$ $(\mathrm{SD} \pm 2.98) . \mathrm{ABH}$ was below $6 \mathrm{~mm}$ in $85.71 \%$ of the patients $(n=12)$. In the other two sites, corresponding to two different patients, initial height was more than $6 \mathrm{~mm}$. However, these patients had undergone previous sinus augmentation for delayed implant placement. Baseline remnant bone height (RBH) considered for these two sites was 10.4 and $12.7 \mathrm{~mm}$, respectively. The implant osteotomy was prepared by means of a trephine (3 $\mathrm{mm}$ internal $/ 4 \mathrm{~mm}$ external diameter) in both cases. Interestingly, although initial $\mathrm{ABH}$ was below $6 \mathrm{~mm}$ in all cases, only three patients underwent maxillary sinus augmentation following a lateral window approach (Fig. 2a). Five sites (33.3\%) were treated with an osteotome technique (Fig. 2b), although only in one case a grafting material was used (Anorganic bovine bone; BioOss ${ }^{\circledR}$, Geistlich Pharma AG, Wolhusen Switzerland), and $46.7 \%$ of the sites did not receive any treatment at all before implant insertion (Fig. 2c). This indicates that $73.3 \%$ of these sites did not receive any augmentation procedure to increase the available bone height prior to implant placement. Implant-supported prostheses included single-tooth restorations $(26.7 \%)$, fixed partial denture $(46.7 \%)$, over- 

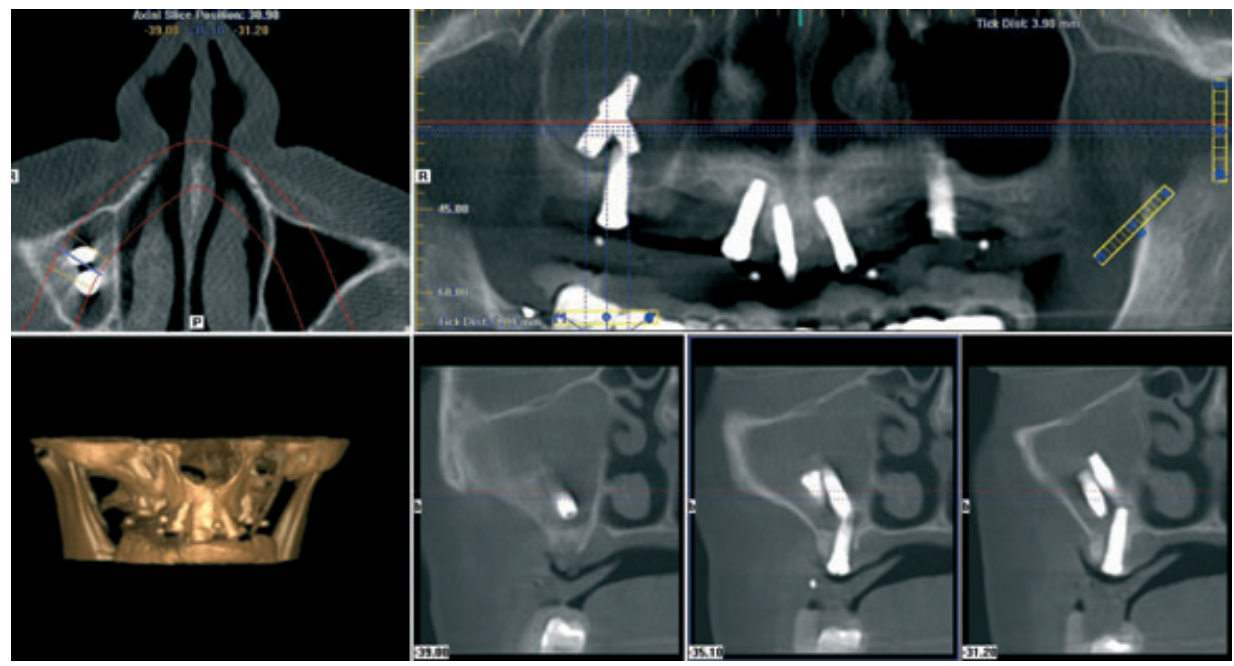

Fig. 1. Two migrations in the same maxillary sinus.

denture $(6.7 \%)$, and full arch rehabilitation $(20.0 \%)$.

Complications associated with the migration were mobility of the fixed prosthetic rehabilitation $(46.7 \%)$, acute sinusitis $(13.3 \%)$, local gingival swelling $(6.7 \%)$, and bacterial or fungal infection $(6.7 \%)$ (Fig. 3). Approximately one-fourth of the patients $(26.7 \%)$ did not report previous symptoms and the diagnoses were incidental, following routine radiographic analysis. Many of our patients (46.7\%) rejected to have the implant removed, given the absence of clinical symptoms. All implants removed $(53.3 \%)$ were extracted using a modified Caldwell Luc approach.

Regarding the frequency distribution of migration several significant results were observed. First, implant design (conical vs. cylindrical) appears to be important for explaining migration of implants, since migration proportion was higher for cylindrical than for conical implants $(P=0.013$ by the binomial test). The practical implications of this result will depend on the a priori probability of each design. Second, it seems that the smaller the implant diameter, the greater the probability of prosthesis mobility $(P=0.01$, chi-square-based randomization test, http://udel.edu/ mcdonald/statrand.xls). Third, there seems to be a significant linear increase of the frequency of mobility as the implant length increases (Kendall Tau- $B=$ 0.536, $P=0.042$ ). This trend is clearly observed when lengths are grouped in intervals of $2 \mathrm{~mm}$ (Kendall Tau- $B=1$ ). Fourth, when $\mathrm{ABH}$ was grouped in $2 \mathrm{~mm}$ intervals, a curvilinear impact on mobility was observed $\left(R^{2}=0.80\right)$, being the interval $5-6.9 \mathrm{~mm}$ worse than the remaining ones. Next, we explored the relationships between variables that may have a clinical impact. In this sense, previous treatment appeared to be associated to the biomaterial used (Cramer $V=0.874$, $P=0.002$ ) and to age (Kendall Tau- $B=-0.41$, $P=0.073$ ). Finally, we tried to determine if we could classify the patients according to whether they had related complications or not. Backward logistic regression $(P$ out $=0.10)$ indicated that the best predictive model included age, $\mathrm{ABH}$, implant diameter, and biomaterial $\left(\chi^{2}(4)=17.39, \quad P=0.002\right.$, Snell-Cox pseudo- $\left.R^{2}=0.70\right)$. All cases were correctly classified as having or not having related complications. As a validation of logistic regression, linear discriminant analysis including these predictors, correctly classified fourteen of the fourteen related complications. Older patients typically require a more complex prosthetic approach, given the higher number of missing teeth, along with poorer bone density, and less quantity of residual bone, which may involve inferior biomechanical conditions in the posterior maxillary bone, as suggested by Regev et al. (1995). The coexistence of this set of factors may facilitate the migration and, subsequently, the prosthetic mobility, which was the most commonly related complication to implant migration (46.7\%). All the descriptive information recorded with the corresponding statistical

\section{Discussion}

Many options are available to rehabilitate atrophic maxillae with implant-supported prostheses. These may include maxillary sinus augmentation, alveolar ridge splitting, horizontal ridge augmentation by means of block grafting or guided bone regeneration (Chiapasco et al. 2006), tilted implants (Tesvalues is presented in Table 1 .
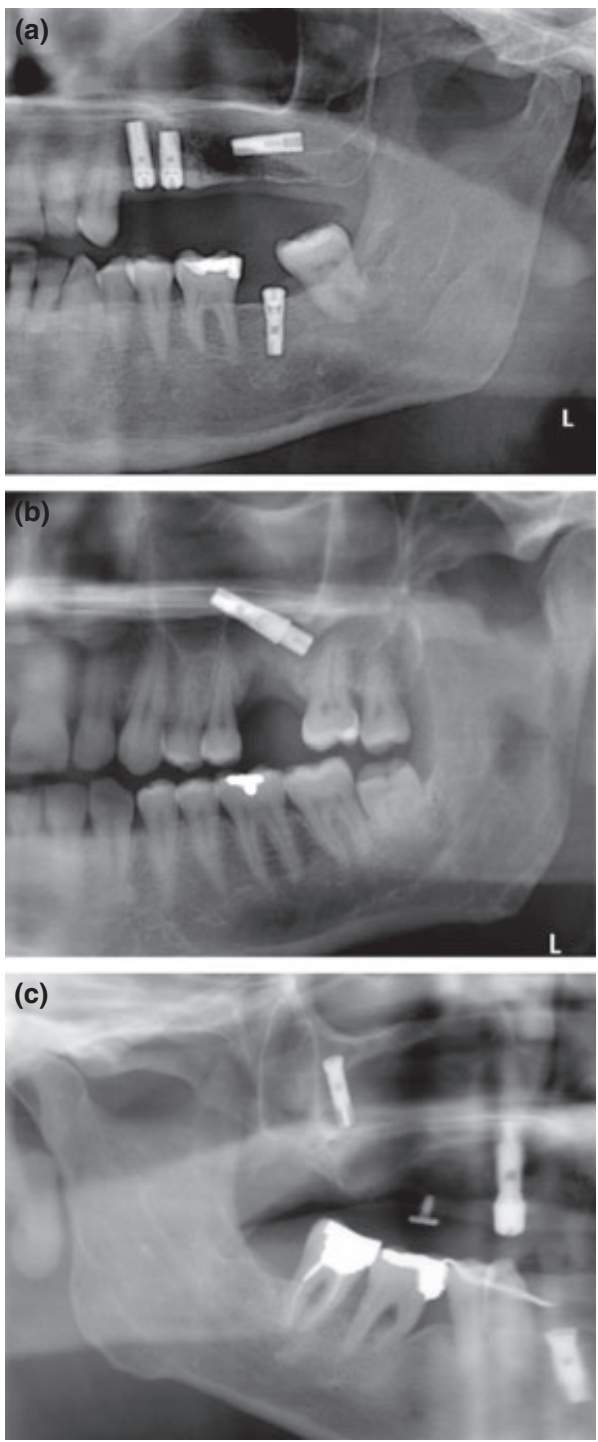

Fig. 2. (a) Implant migration after maxillary sinus augmentation following a lateral window approach. (b) Implant migration after maxillary sinus augmentation following osteotome technique with no grafting. (c) Implant migration in a patient who did not receive any treatment at all before implant insertion.

tori et al. 2008), and zygomatic or pterygoid implants (Malevez et al. 2004). These surgical techniques require advanced training and experience to ensure clinical safety. For example, placement of zygomatic and pterygoid implants requires a learning curve to avoid adverse events, such as ocular lesions, hemorrhage of the pterygoid plexus, oculofacial paraesthesia, or deep fascia infection (Balshi et al. 1999; Penarrocha et al. 2009). Similarly, the success of tilted implants is based on proper case analysis, adequate clinical performance, and the delivery of a welldesigned prosthetic restoration that minimizes lateral occlusal loading (Testori et al. 2008). Sinus augmentation is the most 

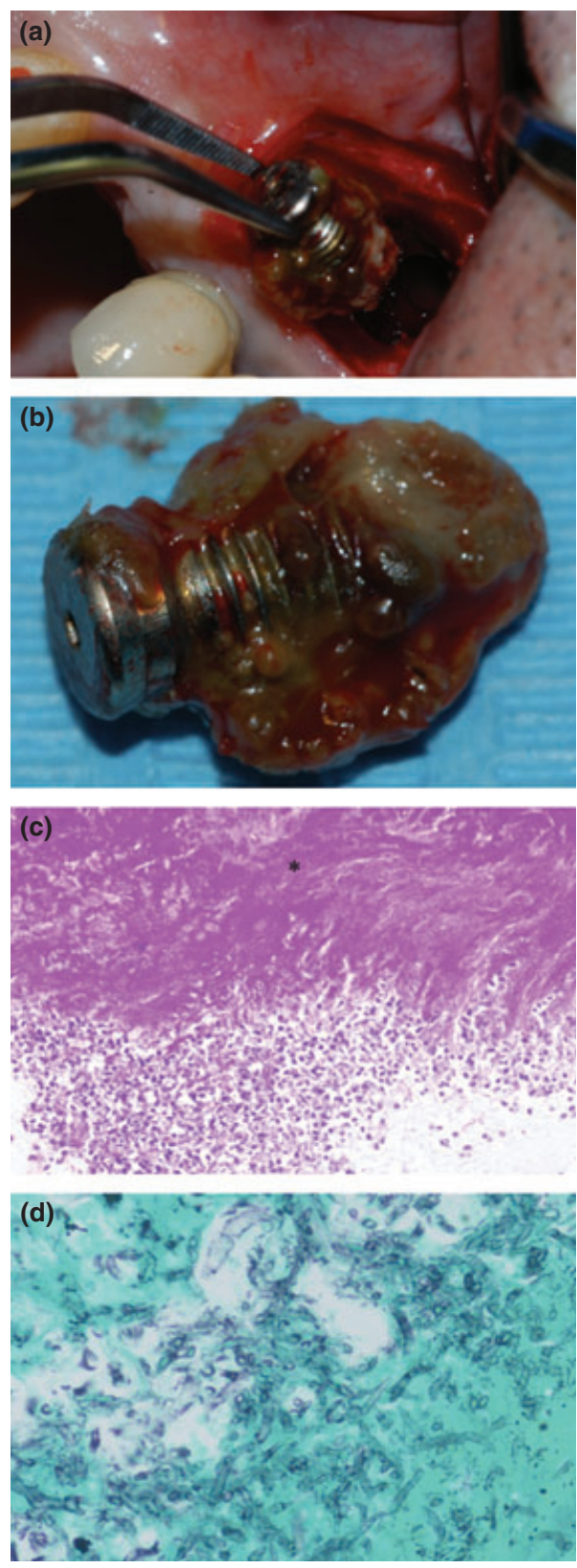

Fig. 3. (a) Lateral approach for migrated implant retrie val. (b) Detail of the implant removed surrounded by unknown material submitted to histopathological anal ysis. (c) Moderate chronic inflammatory reaction in contact with Periodic Acid Schiff positivity substance (*) (PAS $\times 100)$. (d) Demonstration of mycotic ingrowth with numerous hyphae (black color) in the material isolate around dental implant (Grocott's Methenamine Silver Stain $\times 400$ ).

accepted technical approach to compensate for the limited available bone typically present in these locations after tooth loss. Multiple modifications of the original sinus augmentation technique (Boyne \& James 1980) have been proposed, comprising a variety of biomaterials, (Galindo-Moreno et al. 2008) and techniques (e.g. lateral, transcrestal or balloon) (Vitkov et al. 2005; Galindo-Mo- reno et al. 2007). Both the lateral access and the transcrestal osteotome-based approach have demonstrated high predictability, regardless of the grafting material employed, as long as they are applied following an evidence-based approach (Wallace \& Froum 2003; Pjetursson et al. 2008, 2009).

In $80 \%$ of the cases in the here reported study was either performed a sinus augmentation via osteotome approach $(33.3 \%)$ or no augmentation $(46.7 \%)$ at all. Despite the development and predictability of the lateral approach, some clinicians avoid the use of this technique since it may be more traumatic and difficult to perform. Interestingly, for some clinicians this idea could be reinforced, because some authors even have highlighted of placing implants inside the sinus cavity without grafting with similar success rates (Lundgren et al. 2004; Thor et al. 2007). This concept is based on an early study reporting that significant bone gain $(>5 \mathrm{~mm})$ can be achieved even in presence of perforated sinus membrane (Boyne 1993), with no clinical consequences. Conversely, osteotome-based sinus augmentation is considered a less traumatic and safer approach for implant site development in the posterior maxilla.

However, some considerations can be made in this respect. Schneiderian's membrane integrity contributes to adequate graft healing, probably due to its high reparative potential (Srouji et al. 2009, 2010). This element is essential to maintain the sinus cavity isolated from the graft and implant/s. Schneiderian's membrane perforation increases the possibility of complications, such as postoperative maxillary sinusitis due to retrograde bacterial contamination or graft migration into the sinus (Pikos 1999), compromising the success of the technique (Cho et al. 2001), and eventually implant survival (Hernandez-Alfaro et al. 2008). In transcrestal approaches, perforation rates range between $2 \%$ and 25\% (Berengo et al. 2004; Ferrigno et al. 2006). However, perforation of the Schneiderian's membrane cannot be identified unless a simultaneous intraoperative antroscopy is performed (Engelke \& Deckwer 1997). Nkenke and coworkers concluded that a mean elevation of $3.0 \pm 0.8 \mathrm{~mm}$ could be attained by an endoscopically controlled osteotome technique alone before concomitant spontaneous perforation of the sinus membrane in the periphery of the elevated area, occurred (Nkenke et al. 2002). Maximum elevation allowed with no perforation is determined by the elastic properties and thickness of the Schneiderian's membrane, by the strength of its attachment to the sinus floor, by the maxillary sinus anatomy, and by the force applied during the surgical technique (Berengo et al. 2004). During transcrestal sinus floor elevation the force required for membrane detachment increases as the area to elevate does (Pommer \& Watzek 2009). Consequently, in cases of narrow internal sinus anatomy where the circumference of the elevated area is smaller, the elevation height would be higher than in wide sinuses, as long as the same force is applied (Pommer et al. 2009). The area of force transmission applied during sinus elevation by means of osteotomes equals the surface area of the proximal end of the osteotome. Therefore, higher forces are applied using osteotomes of a larger diameter, due to an increased load transfer. Considering that the diameter of the final osteotome used must be similar to the one of the implant, chances of having a membrane perforation may be higher in clinical scenarios in which forces applied are not properly controlled by an experienced clinician. The average height of sinus elevation has been reported to range between 2.5 and $8.6 \mathrm{~mm}$ for transcrestal techniques (Engelke et al. 2003; Toffler 2004; Vitkov et al. 2005; Nedir et al. 2006). This would imply that this procedure might be limited to a residual bone height unless over $8 \mathrm{~mm}$, allowing clinicians to conduct a one-stage surgery protocol (Misch 1990a; Katranji et al. 2008).

Overdrilling, use of trephines, or inadequate performance of an osteotome technique at the time of implant placement in the posterior maxilla could lead to lack of primary implant stability. Insufficient primary stability may induce micromovements in early healing stages, particularly in soft bone. Micromotion is considered an etiologic factor for implant failure. It has been associated with the formation of fibers at the hostimplant interface, as an adaptation to mechanical forces (Akagawa et al. 1986). Continuous micromotion superior to $150 \mu \mathrm{m}$ has been shown to compromise implant healing, while micromotions of $30-50 \mu \mathrm{m}$ are considered acceptable (Pilliar 1991). Davies suggested that micromotion can interfere with formation of the fibrin clot on the implant surface during early wound-healing (Davies 1998). According to Brunski, micromotion can also damage early vascular structures and prevent the chemotaxis of cells needed for bone regeneration, which may result in scar tissue formation instead of bone formation (Brunski 1999). For this reason, early or immediate implant loading has been traditionally avoided during woundhealing period as a prerequisite for osseointe- 


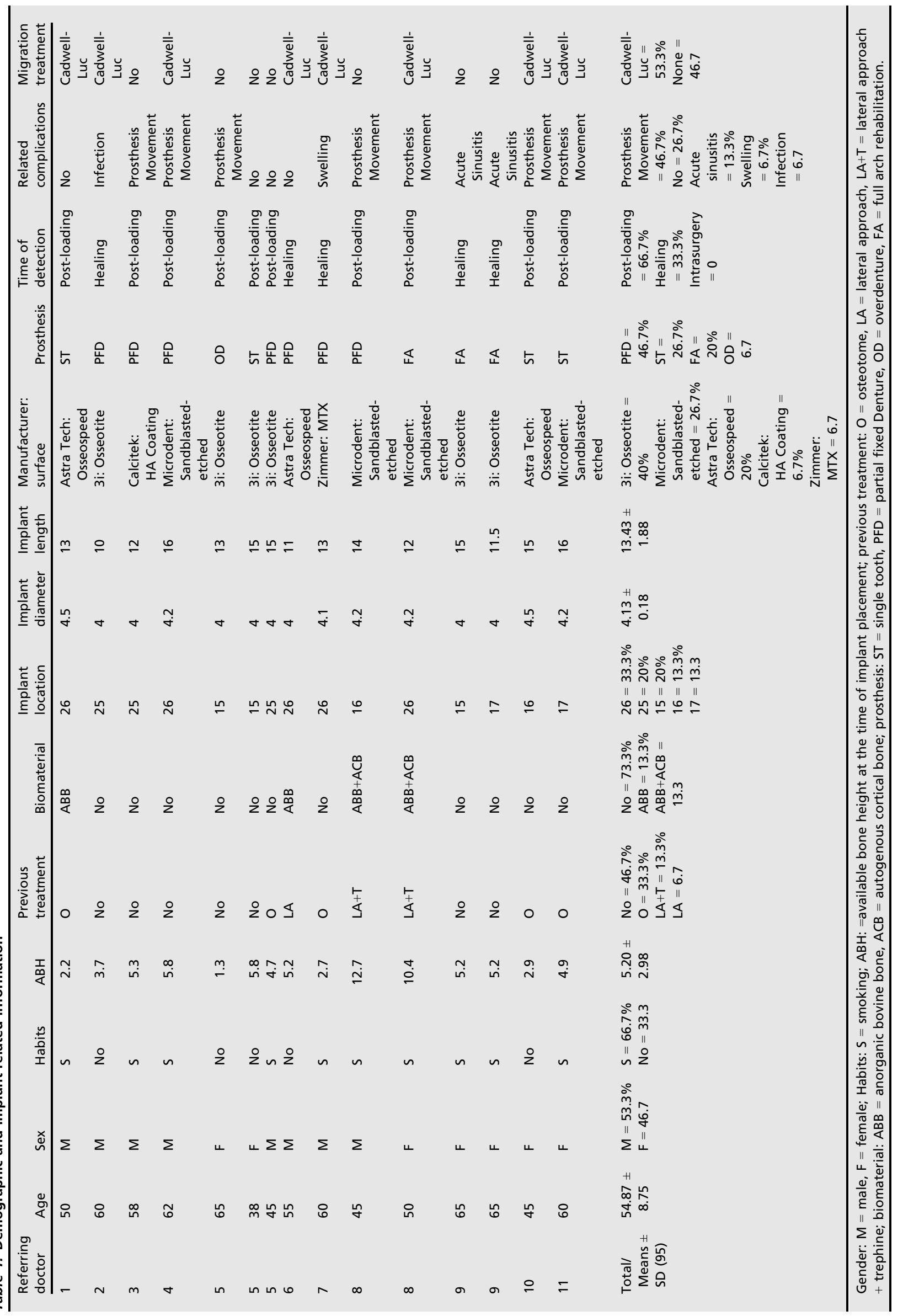


gration (Szmukler-Moncler et al. 1998). This concept is of capital importance in areas of low-density bone, where reasonable doubts regarding implant stability exists. On the other hand, lateral approaches allow us to visualize the new increased ridge where the implant could be stabilized.

Our results showed that the incidence of implant migration into the sinus cavity is higher for cylindrical implants as compared to conical ones, for narrower implants, and when implants were placed in smokers. A singular finding in this study was that the longer the implant, the stronger the association with migration. This could be looked as an illogical result, but we should not disregard that $73.3 \%$ of the sites did not receive any biomaterial and the mean length of the implants in this series was $13.43 \pm 1.88 \mathrm{~mm}$, independently of the mean $\mathrm{ABH} \quad(5.20 \pm$ $2.98 \mathrm{~mm}$ ). Interestingly, mean implant length without bone contact inside the sinus cavity was $8.23 \mathrm{~mm}$. In light of this information, it can be stated that the concordance between the technique conducted by the professionals and the chosen implant for each clinical case was not correct, which could greatly explain the occurrence of this complication for most cases. Another remarkable finding was the statistically significant relationship between $\mathrm{ABH}$ of $5-7 \mathrm{~mm}$ and the increase of migration. According to the literature, this can be considered as the minimal residual bone height necessary to conduct a one-stage sinus augmentation surgery, because primary stability can be achieved (Peleg et al. 1999; Rios et al. 2009; Zinner \& Small 1996). Several classifications discuss the indications for both techniques contemplating a wide array of factors (Misch 1990b; Wang \& Katranji 2008). These concepts may be confusing for non-adequately trained clinicians, which may move them to perform theoretically less invasive procedures or even none.

It is crucial to realize that this emerging complication could be primarily derived from lack of adequate information or knowledge to make a proper clinical judgment and surgical performance. Clinical complications are reported regularly in most journals of the field. From single case reports, to a growing number of larger series, dental implants migrated to paranasal sinuses have been reported over the last 15 years. However, it is a major concern that, in the last few years, several reports including a total of 62 implants migrated to paranasal sinuses have been described (Table 2). In our series, treatment was incorrectly planned for $80 \%$ of the sites. Furthermore, $46.7 \%$ of them did not receive any previous treatment where $\mathrm{RBH}$ was less than $5 \mathrm{~mm}$, ignoring all general recommendations and established protocols. It is important to highlight that of the numer- ous cases previously reported in the literature, just only one patient from the 62 had been treated to properly prepared sites for implant placement, before the migration (Table 2).

Interestingly, Olson and coworkers reported higher survival rates for implants placed in grafted sinus areas than for those placed in maxillary pristine bone /Olson et al. 2000). In this sense, it has been suggested that areas that received maxillary sinus augmentation achieve equal or superior bone volume and density as compared to maxillary pristine bone (Trisi \& Rao 1999; Ulm et al. 1999; Handschel et al. 2009). Our group showed that both cellular activity and vital bone content are higher in areas grafted with a mixture of anorganic bovine bone plus cortical autogenous bone as compared to maxillary pristine bone (Galindo-Moreno et al. 2010). In light of this information, it is reasonable to think that successful maxillary sinus augmentation may prevent implant migration.

Implant placement in atrophic sites commonly requires site development and, therefore, advanced surgical skill and experience to reduce the risk of developing a complication (Wheeler \& Bollinger 2009). In the majority of the cases reported in this study implant placements were performed by general dentists, where proper protocol was

Table 2. Reported cases of migrated dental implants into the maxillary sinus

\begin{tabular}{|c|c|c|c|c|c|c|}
\hline Author & $\begin{array}{l}\text { No. of } \\
\text { patients }\end{array}$ & $\begin{array}{l}\text { No. of } \\
\text { implants } \\
\text { migrated }\end{array}$ & Implant type & $\begin{array}{l}\text { Concomitant/ } \\
\text { Previous } \\
\text { treatment }\end{array}$ & Implant retrieval & $\begin{array}{l}\text { Time of retrieval/detection } \\
\text { after implant insertion }\end{array}$ \\
\hline Borgonovo et al. (2010) & 3 & 3 & Unavailable & None & $\begin{array}{l}\text { Cadwell-Luc/One spontaneously } \\
\text { explanted }\end{array}$ & Unavailable \\
\hline Chiapasco et al. (2009) & 27 & 27 & Straight & None & Cadwell-Luc & 1-24 months \\
\hline Flanagan Flanagan (2009) & 1 & 1 & Tapered & None & Cadwell-Luc & During insertion \\
\hline Galindo et al. (2005) & 2 & 2 & Straight & None & Cadwell-Luc/Follow-up & 4 years/6 months \\
\hline Guler \& Delilbasi (2007) & 2 & 2 & Unavailable & None & Cadwell-Luc & $\begin{array}{l}\text { One during insertion; } \\
\text { One } 8 \text { years later }\end{array}$ \\
\hline lida et al. (2000) & 1 & 1 & Straight & None & Cadwell-Luc & 15 years \\
\hline Kim et al. (2007) & 1 & 1 & Straight & None & Middle meatal antrostomy & 18 months \\
\hline Kitamura (2007) & 1 & 1 & Straight & None & Transnasal endoscope & 3 years \\
\hline Kitamura \& Zeredo (2010) & \multicolumn{6}{|c|}{ Same patient that the previous report } \\
\hline Kluppel et al. (2010) & 2 & 2 & Tapered & None & Cadwell-Luc/Follow-up & 6 months \\
\hline Lubbe et al. (2008) & 1 & 1 & Straight & None & Transnasal endoscope & 3 weeks \\
\hline Nakamura et al. (2004) & 1 & 1 & Tapered & None & Endoscopy & Within days \\
\hline Raghoebar \& Vissink (2003) & 1 & 1 & Straight & None & Cadwell-Luc & 5 months \\
\hline Ramotar et al. (2010) & 2 & 2 & Tapered & None & Endoscopy & Within days \\
\hline Regev et al. (1995) & 3 & 3 & Straight & None & Cadwell-Luc & Months to years \\
\hline Ridaura-Ruiz et al. (2009) & 9 & 9 & Straight & $\begin{array}{l}\text { None/1 } \\
\text { sinus lift }\end{array}$ & $\begin{array}{l}\text { Cadwell-Luc/2 Follow-up/ } \\
1 \text { crestal approach }\end{array}$ & 4-10 months \\
\hline Scarano et al. (2010) & 1 & 1 & Straight & None & Cadwell-Luc & 7 years \\
\hline Tsodoulos et al. (2010) & 1 & 1 & Straight & None & Cadwell-Luc & 8 years \\
\hline Varol et al. (2006) & 3 & 3 & Tapered & None & Endoscopy & Within days \\
\hline TOTAL & 62 & 62 & $\begin{array}{l}48 \text { Straight } \\
9 \text { Tapered } \\
5 \text { Unavailable }\end{array}$ & $\begin{array}{l}1 \text { Sinus lifts } \\
61 \text { non- } \\
\text { previously } \\
\text { treated }\end{array}$ & $\begin{array}{l}47 \text { Cadwell-Luc } \\
8 \text { encoscopy } \\
1 \text { crestal approach } \\
4 \text { followed-up } \\
1 \text { spontaneously expelled } \\
1 \text { middle meatal antrostomy }\end{array}$ & Within days to years \\
\hline
\end{tabular}


not followed since majority of these doctors did not have the advanced training that is required to conduct these sophistical procedures. This can be a problem because articles and course promotional brochures emphasize the simplicity of placing implants by using novel systems, protocols or devices. One clear example is a recent article titled "Technology helps an 'amateur' place implants" (Whitehouse 2008). This type of advertisement encourages an increasing number of dentists, with limited or no surgical training, to perform implant surgical procedures in their practices. Another important factor to consider is that many of the courses on surgical implant placement are sponsored by implant companies, or providers, and are primarily oriented at selling surgical kits and implants. Many of these programs are abbreviated in length, 1-3 days, or less than a week. If minimal educational guidelines could be established and accepted by the implant industry as a whole, most of the abbreviated training courses presently being

\section{References}

Adell, R., Lekholm, U., Grondahl, K., Branemark, P.I., Lindstrom, J. \& Jacobsson, M. (1990) Reconstruction of severely resorbed edentulous maxillae using osseointegrated fixtures in immediate autogenous bone grafts. The International Journal of Oral 巴) Maxillofacial Implants 5: 233-246.

Akagawa, Y., Hashimoto, M., Kondo, N., Satomi, K., Takata, T. \& Tsuru, H. (1986) Initial boneimplant interfaces of submergible and supramergible endosseous single-crystal sapphire implants. Journal of Prosthetic Dentistry 55: 96-100.

Balshi, T.J., Wolfinger, G.J. \& Balshi, S.F. 2nd (1999) Analysis of 356 pterygomaxillary implants in edentulous arches for fixed prosthesis anchorage. The International Journal of Oral et Maxillofacial Implants 14: 398-406.

Berengo, M., Sivolella, S., Majzoub, Z. \& Cordioli, G. (2004) Endoscopic evaluation of the boneadded osteotome sinus floor elevation procedure. International Journal of Oral \&) Maxillofacial Surgery 33: 189-194.

Borgonovo, A., Fabbri, A., Boninsegna, R., Dolci, M. \& Censi, R. (2010) Displacement of a dental implant into the maxillary sinus: case series. Minerva Stomatologica 59: 45-54.

Boyne, P.J. (1993) Analysis of performance of rootform endosseous implants placed in the maxillary sinus. Journal of Long-Term Effects of Medical Implants 3: 143-159.

Boyne, P.J. \& James, R.A. (1980) Grafting of the maxillary sinus floor with autogenous marrow and bone. Journal of Oral Surgery 38: 613-616.

Brunski, J.B. (1999) In vivo bone response to biomechanical loading at the bone/dental-implant interface. Advances in Dental Research 13: 99-119.

Chiapasco, M., Felisati, G., Maccari, A., Borloni, R., Gatti, F. \& Di Leo, F. (2009) The management of taught off the academic environment of the dental schools would be discontinued (Ogunsalu et al. 2009). In summary, to prevent implant migration into the sinus, not only do we need to educate our general dentists partners of the risks associated with implant placement especially in the maxillary posterior area where the bone is typically atrophic and soft in nature, but also to recommend advanced training, cooperation, and to encourage referral and team work (Pikos 2009). These should be the ways to prevent complications, so that we can all benefit from professional interexchange and understanding.

\section{Conclusions}

Implant migration to the maxillary sinus cavity is an increasingly serious complication influenced by multiple factors that involves three main fronts: 1) Implant, 2) Patient and, 3) Surgeon related factors. Understanding that several of these factors are modifiable while others are not, it is our responsibility to identify them to minimize the risk of developing this undesirable complication.

\section{Acknowledgments: The authors} would like to thank Miguel Velasco-Torres, DDS, Dental Radiologist at Centro de Diagnóstico Granada, for his collaboration in the development of this study, Andres Catena for his statistical advices, and Francisco $\mathrm{O}^{\prime}$ Valle for the histopathological support. This study was partially supported by Junta de Andalucia Funding Program for research groups in Spain (Projects \#CTS-138 and \#CTS-583). MPM was also supported by the Talentia Scholarship Program from the Regional Ministry for Innovation, Science and Enterprise - Junta de Andalucia (Spain). Authors do not have any financial interests, either directly or indirectly, in the products listed in the study. complications following displacement of oral implants in the paranasal sinuses: a multicenter clinical report and proposed treatment protocols. International Journal of Oral $\leftrightarrow$ Maxillofacial Surgery 38: 1273-1278.

Chiapasco, M., Zaniboni, M. \& Boisco, M. (2006) Augmentation procedures for the rehabilitation of deficient edentulous ridges with oral implants. Clinical Oral Implants Research 17(Suppl 2): 136-159.

Cho, S.C., Wallace, S.S., Froum, S.J. \& Tarnow, D. P. (2001) Influence of anatomy on Schneiderian membrane perforations during sinus elevation surgery: three-dimensional analysis. Practical Procedures $\oplus$ Aesthetic Dentistry 13: 160-163.

Davies, J.E. (1998) Mechanisms of endosseous integration. International Journal of Prosthodontics 11: 391-401.

Engelke, W. \& Deckwer, I. (1997) Endoscopically controlled sinus floor augmentation. A preliminary report. Clinical Oral Implants Research 8: 527-531.

Engelke, W., Schwarzwaller, W., Behnsen, A. \& Jacobs, H.G. (2003) Subantroscopic laterobasal sinus floor augmentation (SALSA): an up-to-5year clinical study. The International Journal of Oral e) Maxillofacial Implants 18: 135-143.

Ferrigno, N., Laureti, M. \& Fanali, S. (2006) Dental implants placement in conjunction with osteotome sinus floor elevation: a 12-year life-table analysis from a prospective study on 588 ITI implants. Clinical Oral Implants Research 17: 194-205.

Flanagan, D. (2009) A method to retrieve a displaced dental implant from the maxillary sinus. Journal of Oral Implantology 35: 70-74.

Galindo, P., Sanchez-Fernandez, E., Avila, G., Cutando, A. \& Fernandez, J.E. (2005) Migration of implants into the maxillary sinus: two clinical cases. The International Journal of Oral et Maxillofacial Implants 20: 291-295.

Galindo-Moreno, P., Avila, G., Fernandez-Barbero, J.E., Aguilar, M., Sanchez-Fernandez, E., Cutando, A. \& Wang, H.L. (2007) Evaluation of sinus floor elevation using a composite bone graft mixture. Clinical Oral Implants Research 18: $376-$ 382.

Galindo-Moreno, P., Avila, G., Fernandez-Barbero, J.E., Mesa, F., O'Valle-Ravassa, F. \& Wang, H.L. (2008) Clinical and histologic comparison of two different composite grafts for sinus augmentation: a pilot clinical trial. Clinical Oral Implants Research 19: 755-759.

Galindo-Moreno, P., Moreno-Riestra, I., Avila, G., Fernandez-Barbero, J.E., Mesa, F., Aguilar, M., Wang, H.L. \& O'Valle, F. (2010) Histomorphometric comparison of maxillary pristine bone and composite bone graft biopsies obtained after sinus augmentation. Clinical Oral Implants Research 21: $122-128$.

Guler, N. \& Delilbasi, C. (2007) Ectopic dental implants in the maxillary sinus. Quintessence International 38: e238-e239.

Handschel, J., Simonowska, M., Naujoks, C., Depprich, R.A., Ommerborn, M.A., Meyer, U. \& Kubler, N.R. (2009) A histomorphometric metaanalysis of sinus elevation with various grafting materials. Head \&) Face Medicine 5: 12.

Hernandez-Alfaro, F., Torradeflot, M.M. \& Marti, C. (2008) Prevalence and management of Schneiderian membrane perforations during sinus-lift procedures. Clinical Oral Implants Research 19: 91-98.

Iida, S., Tanaka, N., Kogo, M. \& Matsuya, T. (2000) Migration of a dental implant into the maxillary 
sinus. A case report. International Journal of Oral (4) Maxillofacial Surgery 29: 358-359.

Katranji, A., Fotek, P. \& Wang, H.L. (2008) Sinus augmentation complications: etiology and treatment. Implant Dentistry 17: 339-349.

Kim, J.W., Lee, C.H., Kwon, T.K. \& Kim, D.K. (2007) Endoscopic removal of a dental implant through a middle meatal antrostomy. British Journal of Oral e) Maxillofacial Surgery 45: 408409.

Kitamura, A. (2007) Removal of a migrated dental implant from a maxillary sinus by transnasal endoscopy. British Journal of Oral « Maxillofacial Surgery 45: 410-411.

Kitamura, A. \& Zeredo, J.L.. (2010) Migrated maxillary implant removed via semilunar hiatus by transnasal endoscope. Implant Dentistry 19: $16-20$.

Kluppel, L.E., Santos, S.E., Olate, S., Freire Filho, F. W., Moreira, R.W. \& de Moraes, M. (2010) Implant migration into maxillary sinus: description of two asymptomatic cases. Oral et Maxillofacial Surgery 14: 63-66.

Lubbe, D.E., Aniruth, S., Peck, T. \& Liebenberg, S. (2008) Endoscopic transnasal removal of migrated dental implants. British Dental Journal 204: 435436.

Lundgren, S., Andersson, S., Gualini, F. \& Sennerby, L. (2004) Bone reformation with sinus membrane elevation: a new surgical technique for maxillary sinus floor augmentation. Clinical Implant Dentistry \&) Related Research 6: 165173.

Malevez, C., Abarca, M., Durdu, F. \& Daelemans, P. (2004) Clinical outcome of 103 consecutive zygomatic implants: a 6-48 months follow-up study. Clinical Oral Implants Research 15: 18-22.

Misch, C.E. (1990a) Divisions of available bone in implant dentistry. International Journal of Oral Implantology 7: 9-17.

Misch, C.E. (1990b) Classifications and treatment options of the completely edentulous arch in implant dentistry. Dentistry Today 9: 26.

Nakamura, N., Mitsuyasu, T. \& Ohishi, M. (2004) Endoscopic removal of a dental implant displaced into the maxillary sinus: technical note. International Journal of Oral $\oplus$ Maxillofacial Surgery 33: 195-197.

Nedir, R., Bischof, M., Vazquez, L., Szmukler-Moncler, S. \& Bernard, J.P. (2006) Osteotome sinus floor elevation without grafting material: a 1-year prospective pilot study with ITI implants. Clinical Oral Implants Research 17: 679-686.

Nkenke, E., Schlegel, A., Schultze-Mosgau, S., Neukam, F.W. \& Wiltfang, J. (2002) The endoscopically controlled osteotome sinus floor elevation: a preliminary prospective study. The International Journal of Oral \&) Maxillofacial Implants 17: 557-566.

Ogunsalu, C., Judy, K., Obiechina, A., Daisely, H., Bissoon, A.K., Steigmann, M., Dosumu, O., Okojie, V. \& Akeredolu, P. (2009) Systematic approach for the propagation of postgraduate dental implant education in developing countries. Implant Dentistry 18: 249-259.

Olson, J.W., Dent, C.D., Morris, H.F. \& Ochi, S. (2000) Long-term assessment (5-71 months) of endosseous dental implants placed in the augmented maxillary sinus. Annals of Periodontology 5: 152-156.

Peleg, M., Mazor, Z. \& Garg, A.K. (1999) Augmentation grafting of the maxillary sinus and simultaneous implant placement in patients with 3$5 \mathrm{~mm}$ of residual alveolar bone height. The International Journal of Oral e) Maxillofacial Implants 14: 549-556.

Penarrocha, M., Carrillo, C. \& Boronat, A. (2009) Retrospective study of 68 implants placed in the pterygomaxillary region using drills and osteotomes. The International Journal of Oral @) Maxillofacial Implants 24: 720-726.

Pikos, M.A. (1999) Maxillary sinus membrane repair: report of a technique for large perforations. Implant Dentistry 8: 29-34.

Pikos, M.A. (2009) Unhealthy competition. Implant Dentistry 18: 193.

Pilliar, R.M. (1991) Quantitative evaluation of the effect of movement at a porous coated implant bone interface. In: Davies, J.E. ed. The Bone-Biomaterial Interface, 380-387. Toronto: University of Toronto Press.

Pjetursson, B.E., Rast, C., Bragger, U., Schmidlin, K., Zwahlen, M. \& Lang, N.P.. (2009) Maxillary sinus floor elevation using the (transalveolar) osteotome technique with or without grafting material. Part I: implant survival and patients' perception. Clinical Oral Implants Research 20: 667-676.

Pjetursson, B.E., Tan, W.C., Zwahlen, M. \& Lang, N.P.. (2008) A systematic review of the success of sinus floor elevation and survival of implants inserted in combination with sinus floor elevation. Journal of Clinical Periodontology 35: 216240.

Pommer, B., Unger, E., Suto, D., Hack, N. \& Watzek, G. (2009) Mechanical properties of the Schneiderian membrane in vitro. Clinical Oral Implants Research 20: 633-637.

Pommer, B. \& Watzek, G. (2009) Gel-pressure technique for flapless transcrestal maxillary sinus floor elevation: a preliminary cadaveric study of a new surgical technique. The International Journal of Oral ↔) Maxillofacial Implants 24: 817822.

Raghoebar, G.M. \& Vissink, A. (2003) Treatment for an endosseous implant migrated into the maxillary sinus not causing maxillary sinusitis: case report. The International Journal of Oral e) Maxillofacial Implants 18: 745-749.

Ramotar, H., Jaberoo, M.C., Koo Ng, N.K., Pulido, M.A. \& Saleh, H.A. (2010) Image-guided, endoscopic removal of migrated titanium dental implants from maxillary sinus: two cases. Journal of Laryngology et Otology 124: 433-436.

Regev, E., Smith, R.A., Perrott, D.H. \& Pogrel, M. A. (1995) Maxillary sinus complications related to endosseous implants. The International Journal of Oral $\leftrightarrow$ Maxillofacial Implants 10: 451461.

Ridaura-Ruiz, L., Figueiredo, R., Guinot-Moya, R., Pinera-Penalva, M., Sanchez-Garces, M.A., Valmaseda-Castellon, E. \& Gay-Escoda, C. (2009) Accidental displacement of dental implants into the maxillary sinus: a report of nine cases. Clinical Implant Dentistry $\leftrightarrow$ Related Research 11 (Suppl 1): e38-e45.
Rios, H.F., Avila, G., Galindo, P., Bratu, E. \& Wang, H.-L. (2009) The influence of remaining alveolar bone upon lateral window sinus augmentation implant survival. Implant Dentistry 18: 402412.

Scarano, A., Perrotti, V., Carinci, F. \& Shibli, J.A. (2010) Removal of a migrated dental implant from the maxillary sinus after 7 years: a case report. Oral et Maxillofac Surgery, doi: 10.1007/s10006010-0243-8. Epub ahead of print.

Schropp, L., Wenzel, A., Kostopoulos, L. \& Karring, T. (2003) Bone healing and soft tissue contour changes following single-tooth extraction: a clinical and radiographic 12-month prospective study. International Journal of Periodontics $\Theta$ Restorative Dentistry 23: 313-323.

Srouji, S., Ben-David, D., Lotan, R., Riminucci, M., Livne, E. \& Bianco, P. (2010) The innate osteogenic potential of the maxillary sinus (Schneiderian) membrane: an ectopic tissue transplant model simulating sinus lifting. International Journal of Oral e) Maxillofacial Surgery 39: 793-801.

Srouji, S., Kizhner, T., Ben David, D., Riminucci, M., Bianco, P. \& Livne, E. (2009) The Schneiderian membrane contains osteoprogenitor cells: in vivo and in vitro study. Calcified Tissue International 84: 138-145.

Szmukler-Moncler, S., Salama, H., Reingewirtz, Y. \& Dubruille, J.H. (1998) Timing of loading and effect of micromotion on bone-dental implant interface: review of experimental literature. Journal of Biomedical Material Research 43: 192-203.

Tan, W.C., Lang, N.P., Zwahlen, M. \& Pjetursson, B.E. (2008) A systematic review of the success of sinus floor elevation and survival of implants inserted in combination with sinus floor elevation. Part II: transalveolar technique. Journal of Clinical Periodontology 35: 241-254.

Testori, T., Del Fabbro, M., Capelli, M., Zuffetti, F., Francetti, L. \& Weinstein, R.L. (2008) Immediate occlusal loading and tilted implants for the rehabilitation of the atrophic edentulous maxilla: 1 -year interim results of a multicenter prospective study. Clinical Oral Implants Research 19: 227-232.

Thor, A., Sennerby, L., Hirsch, J.M. \& Rasmusson, L. (2007) Bone formation at the maxillary sinus floor following simultaneous elevation of the mucosal lining and implant installation without graft material: an evaluation of 20 patients treated with 44 Astra Tech implants. Journal of Oral (4) Maxillofacial Surgery 65: 64-72.

Toffler, M. (2004) Osteotome-mediated sinus floor elevation: a clinical report. The International Journal of Oral \& Maxillofacial Implants 19: 266-273.

Trisi, P. \& Rao, W. (1999) Bone classification: clinical-histomorphometric comparison. Clinical Oral Implants Research 10: 1-7.

Tsodoulos, S., Karabouta-Voulgaropoulou, I., Voulgaropoulou, M. \& Georgiou, C. (2010) Atraumatic removal of an asymptomatic migrated dental implant into the maxillary sinus: a case report. Journal of Oral Implantology, doi: 10.1563/AAIDJOI-D-10-00053. Epub ahead of print.

Ulm, C., Kneissel, M., Schedle, A., Solar, P., Matejka, M., Schneider, B. \& Donath, K. (1999) Characteristic features of trabecular bone in 
edentulous maxillae. Clinical Oral Implants Research 10: 459-467.

Varol, A., Turker, N., Goker, K. \& Basa, S. (2006) Endoscopic retrieval of dental implants from the maxillary sinus. The International Journal of Oral e) Maxillofacial Implants 21: 801-804.

Vitkov, L., Gellrich, N.C. \& Hannig, M. (2005 Sinus floor elevation via hydraulic detachment and elevation of the Schneiderian membrane. Clinical Oral Implants Research 16: 615-621.
Wallace, S.S. \& Froum, S.J. (2003) Effect of maxillary sinus augmentation on the survival of endosseous dental implants. A systematic review. Annals of Periodontology 8: 328-343.

Wang, H.L. \& Katranji, A. (2008) ABC sinus augmentation classification. International Journal of Periodontics $\oplus$ Restorative Dentistry 28: 383389.

Wheeler, S. \& Bollinger, C.M. (2009) Complication or substandard care? Risks of inadequate implant training Journal of the California Dental Association 37: 647-651.

Whitehouse, J. (2008) Technology helps an "amateur" place implants. Dentistry Today 27: 120, $122,124$.

Zinner, I.D. \& Small, S.A.. (1996) Sinus-lift graft: using the maxillary sinuses to support implants. Journal of the American Dental Association 127: $51-57$. 